\title{
Measuring Quality in Oncology: Challenges and Opportunities
}

Jessica K. DeMartino, PhD

\begin{abstract}
Variations in the quality of cancer care are well documented. A key element of quality monitoring is standardized measures of care. Quality measures may include both process measures and outcome measures. Process measurement is only one aspect of assessing the quality of cancer care; measuring outcomes and providing the appropriate structure for care are also important. A variety of stakeholders, including professional oncology organizations and public and private payors, have developed programs and quality measures to address variations in the delivery of cancer care. To fulfill the current need to explore and discuss how quality is measured in oncology care, NCCN convened the NCCN Oncology Policy Summit: Measuring Quality in Oncology - Challenges and Opportunities. The summit was a forum to discuss current efforts to use quality measures, the value of quality measures, and patient and caretaker perspectives on quality. (JNCCN 2013;11:1482-1491)
\end{abstract}

\section{Executive Summary}

Variations in the quality of cancer care are well documented. ${ }^{1,2}$ A key element of quality monitoring is standardized measures of care. Quality measures may include both process and outcome measures. Process measurement is only one aspect of assessing the quality of cancer care; measuring outcomes and providing the appropriate structure for care are also important. Measuring outcomes such as overall survival can be expensive and complicated by existing comorbidities, and requires significant time before one can appropriately measure the effects on delivery of care. Adherence to process measures is often used as a proxy for long-term survival. The implicit assumption is that adherence to good processes will predict good long-term outcomes. Quality measures can be used for both self-assessment and external review of the quality of care.

Payors and purchasers of health care often use standardized measures to ensure their beneficiaries are re- ceiving high-quality cancer care at a reasonable cost. Both public and private payors are placing a greater emphasis on quality, often by linking reimbursement to quality. In response to this growing trend to demonstrate value, payors, professional organizations, and provider groups have begun to create metrics based on both the literature and established guidelines. Quality measures that quantify the patient experience are also becoming increasingly popular, including length of time away from work or family, speed and completeness of recovery, avoidance of toxicity, and financial hardship. Although all of these factors are important to patients, they may not have equivalent importance to providers or payors who are assessing quality.

To fulfill the current need to explore and discuss how quality is measured in oncology care, NCCN convened the NCCN Oncology Policy Summit: Measuring Quality in Oncology - Challenges and Opportunities in Washington, DC, on Friday, July 12, 2013. Stakeholders gathered to discuss the challenges and opportunities for measuring quality care in oncology. The summit included discussion of current efforts to develop quality measures, the value of quality measures, and patient and caretaker perspectives on quality. The program consisted of short presentations and 3 roundtable discussions with lively dialogue and time for audience questions.

This article explores current efforts and challenges in measuring quality in oncology, and incorporates the discussion and ideas presented at the NCCN Oncology Policy Summit.

\section{Personal Perspectives on Quality - 'How I Define Quality'}

How an individual, whether it is a provider, patient, caregiver, or payor, defines and views quality cancer care often depends on personal experiences. Segments 
of discussion at the summit focused on personal perspectives regarding what constitutes quality and the experiences of patients and caregivers. Panelists focused on what is not considered quality care, including the underuse, misuse, and overuse of care, and care that does not take into consideration the individual patient. The importance was highlighted of patients having access to specialists and care teams with good communication skills and, specifically, care teams who support patient empowerment in decision-making and work to preserve the patient's quality of life. Very simply, in most cases, quality care is the right diagnosis and the right treatment in the right time frame in the right environment.

Several panelists who had previous experience as either a patient or a caregiver discussed their individual experiences and what quality meant to them. Panelists emphasized the importance of listening and communication by all doctors, nurses, and staff with whom patients and their families interact. They discussed how providing the "right" amount of information to patients and their families is a difficult task for physicians and nurses, but is critical to the patient experience. Panelists discussed how the overall culture of a hospital, or how patients and their families are received, all contribute to defining a quality experience. Patients value the basic aspects, such as parking, food, and overall friendliness. Panelists also discussed what they expect of their oncologists. They want acknowledgement of symptoms; understanding from providers that the wait time between testing and hearing the actual diagnosis can be grueling, because every patient assumes they have cancer until informed otherwise; and for providers to understand that although the day may be a routine one for them, it may be one that changes a patient's life forever. Based on their shared experiences, the panelists unanimously agreed that improvements are needed in measuring the patient experience and sharing patient feedback with health care systems to improve quality.

\section{Current Efforts to Measure Quality in Oncology}

Many professional cancer organizations, along with other professional organizations, currently have programs to measure quality in cancer care. These programs vary in the technology used to measure qual- ity, the quality measures used, and who is using the information for what purpose. The following section explores several organizations that were present at the NCCN Oncology Policy Summit and their current efforts to measure and improve quality.

\section{American Society of Clinical Oncology}

ASCO has long been dedicated to quality, the delivery of quality care, and the implementation of quality care globally. ASCO's efforts to develop quality measures, measure quality, and improve care are multipronged.

In 2006, quality measures for breast and colorectal cancer were identified in a collaborative effort between NCCN and ASCO. ${ }^{3}$ Content and methodology experts selected and refined metrics for breast and colorectal cancers based on ASCO's National Initiative on Cancer Care Quality, NCCN measures, and NCCN and ASCO guidelines. Measures were selected based on their impact on disease-free and overall survivals, the degree to which opportunities for improvement existed, and the feasibility of data collection. Using separate processes and methodologies, the American College of Surgeons (ACoS) Commission on Cancer (CoC) developed a similar set of measures for breast and colorectal cancer and submitted them to the National Quality Forum (NQF) for endorsement as part of the NQF Cancer Project. Facilitated by NQF, CoC, ASCO, and NCCN agreed to synchronize their developed measures to ensure that a unified set were presented to the public in April 2007.

ASCO has developed a quality measurement program entitled the Quality Oncology Practice Initiative (QOPI), in which measures are evidence- and consensus-based. ${ }^{4}$ It is a voluntary program that facilitates self-assessment, repetitive quality measurement, and continuous improvement in outpatient oncology care settings. The program, started in 2002, is available to ASCO members for no charge, and most providers are responsible for covering their own costs for data abstraction. Data are collected from retrospective chart reviews and submitted through a secure Web-based application. ASCO has taken this program one step further by certifying practices that participate in QOPI. The QOPI Certification Program evaluates an individual practice's performance in areas that affect patient care and safety, and if scoring requirements are met, the practice receives a 3-year certification that payors, employers, and patients may regard positively. 
ASCO's newest initiative to evaluate quality and subsequently improve care is CancerLinQ. ${ }^{5}$ ASCO's vision for CancerLinQ is to assemble and analyze information from electronic medical record systems in a central knowledge base, which will grow "smarter" over time. Specifically, the system will

- Upload clinical data stored in electronic health records (EHRs) from patients in multiple practices;

- Aggregate information from EHRs, new clinical trials, and published guidelines;

- Identify trends and associations between myriad variables to generate new hypotheses;

- Allow physicians and researchers to evaluate those hypotheses and determine which ones may lead to improved care in real-world settings; and

- Enable clinicians and researchers to quickly apply those conclusions, forming a continuous cycle of learning.

In fall 2013, ASCO will launch its inaugural class of the Quality Training Program. ${ }^{6}$ The purpose of the Quality Training Program is to teach oncology providers to engage in successful quality improvement activities in their practice settings, and to train oncologists to assume quality leadership positions and champion quality initiatives.

\section{National Quality Forum}

The $\mathrm{NQF}^{7}$ is a nonprofit organization founded in 1999 with the primary goal of improving American health care through

- Building consensus on national priorities and goals for performance improvement and working in partnership to achieve them;

- Endorsing national consensus standards for measuring and publicly reporting on performance; and

- Promoting the attainment of national goals through education and outreach programs.

NQF itself does not develop its own measures, and instead might contract development work out to other professional organizations. NQF in turn endorses measures from other organizations using their Consensus Development Process. ${ }^{8}$

NQF has previously endorsed performance measures related to the diagnosis and treatment of various cancers, including breast, lung, prostate, and colon cancers; melanoma; and leukemia. In October 2011,
NQF, at the request of the Department of Health and Human Services, began a 2-phase project focused on identifying, endorsing, and updating a broader set of cancer care performance measures. Specifically, the project sought to endorse measures that focused on hematology and melanoma; prostate, lung, breast, and colon cancers; and palliative care concerns.

Under 2 phases of the cancer endorsement project, NQF endorsed 38 measures suitable for accountability and quality improvement. Of the 38 measures, 28 were previously endorsed and granted continued endorsement status, and 10 were newly submitted measures. In August 2012, the NQF Board of Directors endorsed 22 quality measures addressing cancer care focused on conditions such as leukemia, prostate cancer, and multiple myeloma. ${ }^{9}$ Care delivery measures include radiation dose limits, hospice readmissions, and care planning. Notably, 5 measures maintaining endorsement concentrate on palliative care, an increasingly important area for quality measurement and improvement. The measures deal with overtreatment of terminally ill patients, specifically addressing patient preferences and use of services at the end of life.

Every 3 years, endorsed measures are reevaluated against criteria and are reviewed alongside newly submitted (but not yet endorsed) measures. This head-to-head comparison of new and previously endorsed measures fosters harmonization and helps ensure that NQF is endorsing the best available measures.

\section{Commission on Cancer}

The ACoS CoC is a consortium of professional organizations dedicated to improving survival and quality of life for patients with cancer through standardsetting, prevention, research, education, and the monitoring of comprehensive quality care. More than $70 \%$ of all patients with newly diagnosed cancer are treated in the more than 1500 CoC-accredited cancer programs nationwide. ${ }^{10}$

The CoC Accreditation Program encourages hospitals, treatment centers, and other facilities to become $\mathrm{CoC}$ accredited. There are currently 9 categories of accreditation cancer programs can be labeled. ${ }^{11}$ Cancer Program Standards 2012: Ensuring Patient-Centered Care established new requirements around patient-centered needs and expanded the focus on improving the quality of care and patient outcomes. 
Following are 5 key elements of success for a CoC-accredited cancer program:

- The clinical services provide state-of-the-art pretreatment evaluation, staging, treatment, and clinical follow-up for patients with cancer seen at the facility for primary, secondary, tertiary, or end-of-life care.

- The cancer committee leads the program through setting goals, monitoring activity, evaluating patient outcomes, and improving care.

- The cancer conferences provide a forum for patient consultation and contribute to physician education.

- The quality improvement program is the mechanism for evaluating and improving patient outcomes.

- The cancer registry and database is the basis for monitoring the quality of care.

The National Cancer Data Base (NCDB), a joint program of $\mathrm{CoC}$ and the American Cancer Society, is a nationwide oncology outcomes database for more than $1500 \mathrm{CoC}$-accredited cancer programs in the United States and Puerto Rico. ${ }^{12}$ Approximately $70 \%$ of all newly diagnosed cancer cases in the United States are captured at the institutional level and reported to the NCDB. The NCDB, begun in 1989, now contains approximately 29 million records from hospital cancer registries across the United States. Data on all types of cancer are tracked and analyzed. Data elements are collected and submitted to the NCDB from CoC-accredited cancer program registries using nationally standardized data item and coding definitions and includes patient characteristics, cancer staging and tumor histologic characteristics, type of first-course treatment administered, and outcomes information. These data are used to explore trends in cancer care, to create regional and state benchmarks for participating hospitals, and to serve as the basis for quality improvement.

The Web-based Cancer Program Practice Profile Reports $\left(\mathrm{CP}^{3} \mathrm{R}\right)$ offer local providers comparative information to assess adherence to and consideration of standard of care therapies for major cancers. ${ }^{13}$ This reporting tool provides a platform from which to promote continuous practice improvement to improve quality of patient care at the local level, and also permits hospitals to compare their care for these patients relative to that of other providers. The goal is to empower clinicians, administrators, and other staff to work cooperatively and collaboratively to identify problems in practice and delivery and to implement best practices that will diminish disparities in care across CoC-accredited cancer programs.

\section{National Committee for Quality Assurance}

The National Committee for Quality Assurance (NCQA) is a private, 501(c)(3) not-for-profit organization dedicated to improving health care quality. ${ }^{14}$ Since its founding in 1990, NCQA has been a central figure in driving improvement throughout the health care system, helping to elevate the issue of health care quality to the top of the national agenda.

NCQA's programs and services reflect a straightforward formula for improvement: measure, analyze, improve, repeat. NCQA makes this process possible in health care by developing quality standards and performance measures for a broad range of health care entities. These measures and standards are the tools that organizations and individuals can use to identify opportunities for improvement.

NCQA primarily focuses on accreditation, certification, and recognition of various sectors of the health care industry. NCQA has clinician recognition for areas such as diabetes and cardiovascular care, and report cards on payors regarding the quality of their contracted physicians and hospitals.

In March 2013, NCQA extended concepts of the patient-centered medical home by launching a new evaluation program for specialists outside of primary care: NCQA Patient-Centered Specialty Practice (PCSP) Recognition. ${ }^{15}$ The new program is modeled on NCQA's Patient-Centered Medical Home Recognition program, the most widely adopted medical home model in the country. Under PCSP, specialty practices committed to improving access, communication, and care coordination can earn accolades as the "neighbors" that surround and inform the medical home and colleagues in primary care.

John Sprandio, MD, and his southeast Pennsylvania oncology practice, Consultants in Medical Oncology and Hematology $(\mathrm{CMOH})$, are early adopters of this program and are pursuing PCSP recognition. Other oncology groups are also considered early adopters of the program.

\section{Community Oncology Alliance}

The mission of the Community Oncology Alliance (COA) is to protect and foster the community on- 
cology delivery system in the United States through public policy, advocacy, and education. ${ }^{16}$ Because most Americans battling cancer receive treatment in the community setting, ensuring the vitality of the community cancer care delivery system is imperative for patient well-being.

A major COA initiative underway for the past year has been the development and implementation of the oncology medical home model. ${ }^{17}$ Through development and evolution of the oncology medical home model, COA wants to provide oncology providers with a care model that can enhance the quality of cancer care while controlling overall costs in a system in which quality and value are measured and continuously improved.

COA intends to offer, without any commercial interest, a model that allows practices to become oncology medical homes. This will be accomplished through providing practices with a 3-step approach that allows them to move along a trajectory of increasing thoroughness toward becoming a medical home. Some of the specific tasks involved in realizing this overall goal are as follows:

- Defining a core set of standardized quality and value measures to document performance

- Developing a benchmark capability that allows providers to compare performance against their peers in a systematic and highly efficient manner

- Developing a set of services and tools, including information and materials, that provide a stepped approach for providers to move along the trajectory of becoming a fully functioning medical home

- Establishing a forum of information exchange for practices to continually improve processes and outcomes

- Developing different payment models and contracts with Medicare and private payors to make the medical home a viable model for oncology

The Board of Directors of COA established a steering committee to direct the overall efforts of the COA oncology medical home initiative. The committee is composed of oncologists, private payors, practice administrators, a Doctor of Pharmacy, a patient, and industry and cancer community group representation. To date, the committee has helped define the model of the oncology medical home, starting with identifying the needs of patients, pro- viders, and payors in the delivery of cancer care. This effort then allowed the committee to identify and endorse an initial set of 16 quality and value measures of cancer care in July 2012. ${ }^{18}$ The initial list covers adherence to NCCN Clinical Practice Guidelines in Oncology; decreasing emergency room visits and inpatient admissions; screening and interventions for psychosocial needs; use of hospice services; and patient satisfaction. Additionally, the committee backed the development of a patient satisfaction tool, which is a modification of the Consumer Assessment of Healthcare Providers and Systems (CAHPS) survey tool.

An implementation team was also formed to identify the information, tools, and software required to transform oncology centers into fully functioning oncology medical homes. To date, the team, composed of oncology practice administrators, has identified the resources ( $>50$ and counting) and is working to pull those together into an oncology medical home "tool kit." The goal is to provide practices, according to an oncology medical home assessment, with a customized set of tools to move along a path of increasing sophistication.

\section{Consultants in Medical Oncology and Hematology}

Dr. John Sprandio has engineered his practice, $\mathrm{CMOH}$, into an oncology patient-centered medical home (OPCMH). This was accomplished after many years and changes to the practice, including a reengineering of the process of care, including a nurse triage symptom algorithm system; imbedding information technology functionality into an existing EHR system; and ensuring physician buy-in and compliance. $\mathrm{CMOH}$ was able to reduce the incidence of hospitalizations and emergency room visits, reduce the use of imaging services and other diagnostic tools, better coordinate services with other care practitioners, and improve communication with patients regarding their care, especially palliative and hospice care options. ${ }^{19}$ According to CMOH's estimates based on internal data, the OPCMH model of care has saved $\$ 1$ million per physician per year. One of the greatest challenges around implementation of this type of care model is securing appropriate reimbursement from payors. $\mathrm{CMOH}$ has NCQA recognition as Level 3 Patient-Centered Medical Home. Perspective adopters of this model should contact payors to discuss development of shared-savings programs, pay 
for performance/value programs, and other payment methodologies that reward practices financially for improving quality and reducing costs and use.

\section{Come Home Project}

Barbara McAneny, MD, CEO of New Mexico Cancer Center, received a $\$ 19.8$ million grant from the Medicare Innovation Center as part of its Health Care Innovation awards. This grant will use the patient care policies created by New Mexico Cancer Center and implement them at 6 other practices in the Community Oncology Medical Home, "Come Home," program.

Because of the complicated, expensive, and often fragmented nature of cancer care, there are often suboptimal outcomes, high cost, and patient dissatisfaction with care. The Come Home program addresses these issues by focusing on team-based care, which is directed by physicians, and provides patients with seamless, integrated, high-quality cancer care and has been referred to as an oncology physician-directed medical home. Through comprehensive outpatient oncology care, including patient education, team care, medication management, and 24/7 practice access and inpatient care coordination, the medical home model will improve the timelines and appropriateness of care, reduce unnecessary testing, and reduce avoidable emergency room visits and hospitalizations. Through avoiding duplication of care and providing patient education and significant support services, this project is expected to help lead the way for the next generation of cancer care.

\section{Summary of Panelists' Thoughts on Current Efforts to Measure Quality in Oncology}

Much discussion during the roundtables focused on current efforts to measure quality in oncology. As can be seen in the earlier descriptions of current efforts to measure quality in oncology, organizations are taking varied approaches in measuring quality, improving care, and making care more cost-efficient. Panelists agreed on several issues that face all stakeholders attempting to measure quality in oncology. Because of the complexity of the diseases that constitute cancer, data are more complex, and in many cases evidence is not clear and strong. These attributes lead to quality being more difficult to measure in oncology than other clinical areas. Stakeholders must develop more evolved paradigms to deal with these complex patients and data, and ideally real-time feedback of data would be central to these systems.

Panelists also agreed about the importance of EHRs to measuring quality. According to panelists, current EHR systems function like filing cabinets and face many interoperability issues. EHRs must be enhanced to fully capture their potential to measure quality and improve care. They also must be improved to match the workflow of oncology practice for better incorporation into medical practice.

Panelists expect documentation of guideline or pathway adherence to become standard procedure across all oncology practices. This will allow quality measurement programs to assess dimensions that matter more to patients and their families. It was also thought that this could allow the development of quality measures that go beyond basic process measures that often assess what are considered very basic standards of care that everyone should be performing automatically.

Oncologists are the scarcest resource in the practice of oncology care, and therefore should be focused on work only oncologists can perform. Oncologists should not be spending their time as data analysts or data enterers. Systems must be implemented that can help physicians move along the pathway to generate better quality in the environment in which they practice. The oncology care system must determine which quality measurement programs are practical on a national scale, and which programs are more suitable to local implementation. Will a one-size-fits-all approach to measuring quality work in oncology, or will a more piecemeal approach better suit the oncology community?

\section{Public and Private Payors Quality Measurement Programs}

Public and private payors are initiating their own quality measurement programs to ensure their beneficiaries are receiving the best care at a reasonable cost. Many payors are tying quality measurement to reimbursement, incentive payments, or penalties. The following section will examine payors who were present at the NCCN Oncology Policy Summit, and their current efforts to measure and improve quality.

\section{Centers for Medicare and Medicaid Services}

The Centers for Medicare and Medicaid Services (CMS) has a variety of quality reporting and perfor- 
mance programs. The programs include hospital quality reporting, physician quality reporting, post-acute care and other setting quality reporting, payment model reporting, and population quality reporting. The measure domains include clinical care, care coordination, population/community health, efficiency and cost reduction, safety, and patient-centered experience and outcomes. The quality programs measure and improve care at the community level, the practice setting level, and the individual physician level.

Within Medicare, quality measurement is very stovepiped and separate, with different programs and measures for entities such as physicians, Prospective Payment System (PPS)-exempt cancer hospitals, acute care hospitals, hospice, and Accountable Care Organizations. In many ways, CMS has rudimentary measures that are not really capable of discerning variation and performance. CMS has recognized these issues and has developed a vision for performance measurement that would make the system more cohesive. ${ }^{20}$ Their vision includes aligning measures with the National Quality Strategy ${ }^{21}$ and current measure domains, implementing measures that fill critical gaps, aligning measures across programs when appropriate, leveraging opportunities to align with the private sector, and focusing on patientcentered measures, including patient outcomes and experience. In addition, CMS would like to focus on core sets of measures and measure concepts, maintain optional measures that can be applied to specialties, and remove measures that are no longer appropriate or needed, all while maximizing improvement in quality and minimizing provider burden.

The Affordable Care Act (ACA) established a quality reporting program for PPS-exempt cancer hospitals $(\mathrm{PCH})$ that will require reporting of quality measures of process, structure, outcome, patients' perspective on care, efficiency, and costs of care on the CMS Web site. Five quality measures for the fiscal year 2014 program and subsequent years were finalized in 2013.22 All 5 measures are NQF-endorsed measures and include 3 cancer-specific process-ofcare measures:

- NQF \#0559: Combination chemotherapy is considered or administered within 4 months (120 days) of diagnosis for women younger than 70 years with AJCC T1c,NO,M0, or stage II or III hormone receptor-negative breast cancer.

- NQF \#0220: Tamoxifen or third-generation aro- matase inhibitor is considered or administered within 1 year (365 days) of diagnosis for women with AJCC T1c,N0,M0, or stage II or III hormone receptor-positive breast cancer.

- NQF \#0223: Adjuvant chemotherapy is considered or administered within 4 months (120 days) of diagnosis for patients younger than 80 years with AJCC stage III (lymph node-positive) colon cancer.

This program will continue to add NQF-endorsed quality measures, with 1 new measure added in fiscal year (FY) 2015 and 13 new measures added in FY $2016 .{ }^{23}$ Of the 13 proposed measures for FY 2016, 6 are for the Surgical Care Improvement Project, 6 are clinical process/oncology care measures, and the last measure is a Patient Experience of Care measure (the Hospital Consumer Assessment of Healthcare Providers and Systems survey).

An additional CMS quality measurement program that may affect oncology is the Physician Quality Reporting System (PQRS). ${ }^{24}$ Some program changes were contained in the ACA, including payment penalties starting in 2015 based on 2013 performance. ${ }^{25}$ The physicians who elect not to participate or are found unsuccessful during the 2013 program year will receive a $1.5 \%$ payment penalty. The 2013 PQRS program will include 259 quality measures, of which approximately 30 are cancerrelated. An eligible professional may choose from the following methods to submit data to CMS: claimsbased, registry-based, qualified EHR, or the Group Practice Reporting Option.

CMS has initiatives to improve the quality of care provided to Medicaid enrollees in clinical areas such as dental care, obesity, maternal and infant health, vaccines, and prevention. The ACA requires the development of a core set of health quality and performance measures for adults to determine the quality of care provided to enrollees. ${ }^{26}$ Most likely, very few of these measures will relate to oncology care, but it is a step in the right direction for Medicaid enrollees. It also provides federal funding to increase primary care payment rates and for states that provide recommended adult preventive services and vaccinations. Quality reporting within Medicaid presents different challenges from that within Medicare. Because Medicaid is a state-run program, the quality and depth of data vary greatly, with some states leading the way in developing quality report- 
ing, whereas other states struggle just to collect basic data. The largest challenge is ensuring Medicaid staff have a uniform picture of quality across all states, along with being able to adequately and uniformly measure quality.

\section{Blue Cross Blue Shield of Michigan}

Blue Cross Blue Shield of Michigan's (BCBSM) program, Value Partnerships, is a collection of clinically oriented initiatives that are significantly improving the quality of patient care throughout Michigan. ${ }^{27}$ Through Value Partnerships, BCBSM works collaboratively with physicians, physician organizations, and most of the acute-care hospitals in the state to improve the health care provided to all Michigan residents. The stated goals of the program are to enhance clinical quality, decrease complications, manage costs, eliminate errors, and improve health outcomes.

In 2007, as part of its Value Partnerships program, BCBSM began reimbursing oncology practices for data collection costs associated with their participation in QOPI, and the number of participating practices increased from 3 to $36 .^{28}$ After 2 years of data collection, the Michigan Oncology Quality Consortium was founded to look at medical practice characteristics and how the practices' adherence to processes was related to valuable patient outcomes. They looked at adherence in 4 areas: core processes, processes specific to a particular cancer, processes related to supportive care, and end-of-life processes. Three areas were identified with generally low scores where improvement recommendations could be made. The researchers found that collecting data and using them to show physicians their adherence, or lack thereof, to quality processes was not sufficient to measurably improve quality.

Another aspect of BCBSM's Value Partnership program is the Hospital Collaborative Quality Initiatives program. ${ }^{29}$ Hospital Collaborative Quality Initiatives are programs that address some of the most common and costly areas of surgical and medical care. Multiple facilities collect and share data to improve the delivery and quality of care. Data are collected for all patients, not just those covered by BCBSM. Collaborative Quality Initiatives permit a more robust analysis of the link between processes and outcomes of care than can be achieved through examining one group or institution. Two Collaborative Quality Initiatives focus on oncology care: the Michigan Breast
Oncology Quality Initiative and the Michigan Radiation Oncology Quality Initiative.

BCBSM supports these programs financially by giving providers money to help develop infrastructure, such as registries to objectively look at the care that is provided and the outcomes, and to identify opportunities for improvement on both the process and outcomes sides of care. BCBSM does not receive individual data, but might receive aggregated data to examine. They then have the opportunity to compare aggregate data to claims data to show the value of these programs to customers.

\section{Employer Perspectives on Quality}

The employer perspective on quality was also discussed at the summit. For many employers, the 2 most important aspects of quality care is getting employees to the "right" provider and back to work in a timely fashion. Panelists discussed the importance of transparency in quality data so patients and their employers know where to go for care and how much they should be paying. The impact of copayments and coinsurance on employees was highlighted. Sometimes copays need to be adjusted to get employees to be compliant with recommended care. Compliance in turn saves money on emergency room visits and hospitalizations and allows employees to return to work sooner. A question was asked of the panelists as to how do you ensure employees and beneficiaries are receiving quality care. The answers from the panelists were not overwhelmingly reassuring, ranging from this cannot be ensured at this point, to relying on payors to help dictate, to it sometimes being easier to tell a patient where not to receive care.

\section{Conclusions}

Measuring quality in oncology is a complex, multidimensional challenge. Although the number of organizations measuring quality and the number of quality measures are increasing, many obstacles remain, including the ability to actually impact and improve care within the current systems and measures. Questions remain as to whether current measures are granular and specific enough to allow differentiation between high and low quality care and providers. Can an "A" provider be distinguished from a "C" provider?

The quality of the data being collected is also debated. The complexity of cancer, the variety of treat- 
ment options, and the ever-increasing personalization of care leads to complex data that in many cases are not clear or strong. Many stakeholders would argue that "better" quality data are needed to inform the development of quality measures that go beyond basic process measures and can provide feedback to better care. Improvements in the collection of patient-reported outcomes, better measures to gauge the patient experience, and more prospective quality measurement are needed. Along with variability in the quality of data is the great increase in data available from electronic health and medical records that must be incorporated into the practice of oncology.

Ultimately, stakeholders must determine if today's quality measurement systems and measures are adequate to truly address disparities in the quality of oncology care patients receive. What changes are needed to improve care and decrease costs? Can the various programs and measures be brought together in a cohesive way that will cut down on redundancies and wastes of financial and clinical resources? The oncology community, including patients, providers, and manufacturers, needs quality measurement systems that can truly improve care for all patients.

\section{References}

1. Bilimoria KY, Raval MV, Bentrem DJ, et al. National Assessment of Melanoma Care Using Formally Developed Quality Indicators. J Clin Oncol 2009;27:5445-5451.

2. Spencer BA, Miller DC, Litwin MS, et al. Variations in quality of care for men with early-stage prostate cancer. J Clin Oncol 2008;26:3735-3742.

3. Desch CE, McNiff KK, Schneider EC, et al. American Society of Clinical Oncology/National Comprehensive Cancer Network quality measures. J Clin Oncol 2008;26:3631-3637.

4. Neuss MN, Desch CE, McNiff KK, et al. A process for measuring the quality of cancer care: the Quality Oncology Practice Initiative. J Clin Oncol 2005;23:6233-6239.

5. CancerLinQ. ASCO Web site. Available at: http://www.asco.org/ institute-quality/cancerlinq. Accessed August 20, 2013.

6. ASCO Quality Training Program. ASCO Web site. Available at: http://www.asco.org/institute-quality/asco-quality-trainingprogram. Accessed August 20, 2013.

7. National Quality Forum. Available at: http://www.qualityforum. org/Home.aspx. Accessed August 9, 2013.

8. Consensus Development Process. National Quality Forum Web site. Available at: http://www.qualityforum.org/Measuring Performance/Consensus_Development_Process.aspx. Accessed August 9, 2013.

9. NQF Endorses Cancer Measures. National Quality Forum Web site. Available at: http://www.qualityforum.org/News_ And_Resources/Press_Releases/2012/NQF_Endorses_Cancer_ Measures.aspx. Accessed August 20, 2013.
10. About the CoC. American College of Surgeons Web site. Available at: http://www.facs.org/cancer/coc/cocar.html. Accessed August 20, 2013

11. Categories of Accreditation. American College of Surgeons Web site. Available at: http://www.facs.org/cancer/coc/categories.html. Accessed August 20, 2013.

12. National Cancer Data Base. American College of Surgeons Web site. Available at: http://www.facs.org/cancer/ncdb/index.html. Accessed August 20, 2013.

13. Cancer Program Practice Profile Reports $\left(C P^{3} R\right)$. American College of Surgeons Web site. Available at: http://www.facs.org/ cancer/ncdb/cp3r.html. Accessed August 20, 2013.

14. About NCQA. NCQA Web site. Available at: http://www.ncqa. org/AboutNCQA.aspx. Accessed August 9, 2013

15. New NCQA Program Extends Medical Home Concepts Beyond Primary Care. NCQA Web site. Available at: http://www.ncqa. org/Newsroom/2013NewsArchives/NewsReleaseMarch252013. aspx. Accessed August 9, 2013.

16. About the Community Oncology Alliance. Community Oncology Alliance Web site. Available at: http://www.communityoncology. org/site/about-coa.htm. Accessed August 9, 2013.

17. Oncology Medical Home. Available at: http://www. medicalhomeoncology.org/. Accessed August 9, 2013

18. COA Oncology Medical Home Committee approves 16 measures to evaluate and reward quality, cost efficiency and outcomes. PRNewswire Web site. Available at: http://www.prnewswire.com/ news-releases/coa-oncology-medical-home-committee-approves16-measures-to-evaluate-and-reward-quality-cost-efficiency-andoutcomes-162846706.html. Accessed August 9, 2013.

19. Profile: adapting patient-centered medical home principles and tools for an oncology practice. Available at: http://www.ncqa org/portals/0/programs/recognition/Quality\%20Profiles-\%20 PCMH\%20ONC\%20FINAL.pdf. Accessed August 9. 2013.

20. Goodrich K. Quality measurement strategy and alignment. Available at: http://www.ama-assn.org/resources/doc/cqi/pcpi032912-goodrich.pdf. Accessed August 9, 2013.

21. Department of Health \& Human Services. Report to Congress: National strategy for quality improvement in health care. March 2011. Available at: http://www.ahrq.gov/workingforquality/nqs/ nqs2011annlrpt.pdf. Accessed August 9, 2013.

22. Medicare program; hospital inpatient prospective payment systems for acute care hospitals and the long-term care hospital prospective payment system and proposed fiscal year 2014 rates; quality reporting requirements for specific providers; hospital conditions of participation. Fed Regist 2013;78:27710-27720.

23. Medicare program; hospital inpatient prospective payment systems for acute care hospitals and the long-term care hospital prospective payment system and fiscal year 2014 rates; quality reporting requirements for specific providers; hospital conditions of participation; payment policies related to patient status. Fed Regist 2013;78:50495-51040.

24. Physician Quality Reporting System. CMS.gov. Available at: http:// www.cms.gov/Medicare/Quality-Initiatives-Patient-AssessmentInstruments/PQRS/index.html. Accessed August 29, 2013.

25. Physician quality measure reporting. American Medical Association Web site. Available at: http://www.ama-assn.org/ ama/pub/physician-resources/clinical-practice-improvement/ clinical-quality/physician-quality-reporting-system.page. Accessed August 29, 2013. 
Measuring Quality in Oncology

26. Quality of care \& delivery systems. Medicaid.gov. Available at: http://www.medicaid.gov/AffordableCareAct/Provisions/Qualityof-Care-and-Delivery-Systems.html. Accessed August 29, 2013.

27. Value Partnerships overview. Blue Cross Blue Shield Blue of Michigan Web site. Available at: http://www.bcbsm.com/ providers/value-partnerships/value-partnerships-overview.html. Accessed September 3, 2013.
28. Blayney DW, Severson J, Martin CJ, et al. Michigan oncology practices showed varying adherence rates to practice guidelines, but quality interventions improved care. Health Aff (Millwood) 2012;31:718-728.

29. Hospital Collaborative Quality Initiatives. Blue Cross Blue Shield Blue of Michigan Web site. Available at: http://www. bcbsm.com/providers/value-partnerships/collaborative-qualityinitiatives.html. Accessed September 3, 2013. 